\title{
Congenital cytomegalovirus infection
}

\author{
Citomegalovirose congênita
}

José Ricardo Dias Bertagnon ${ }^{1}$, Sarah Rossi ${ }^{1}$

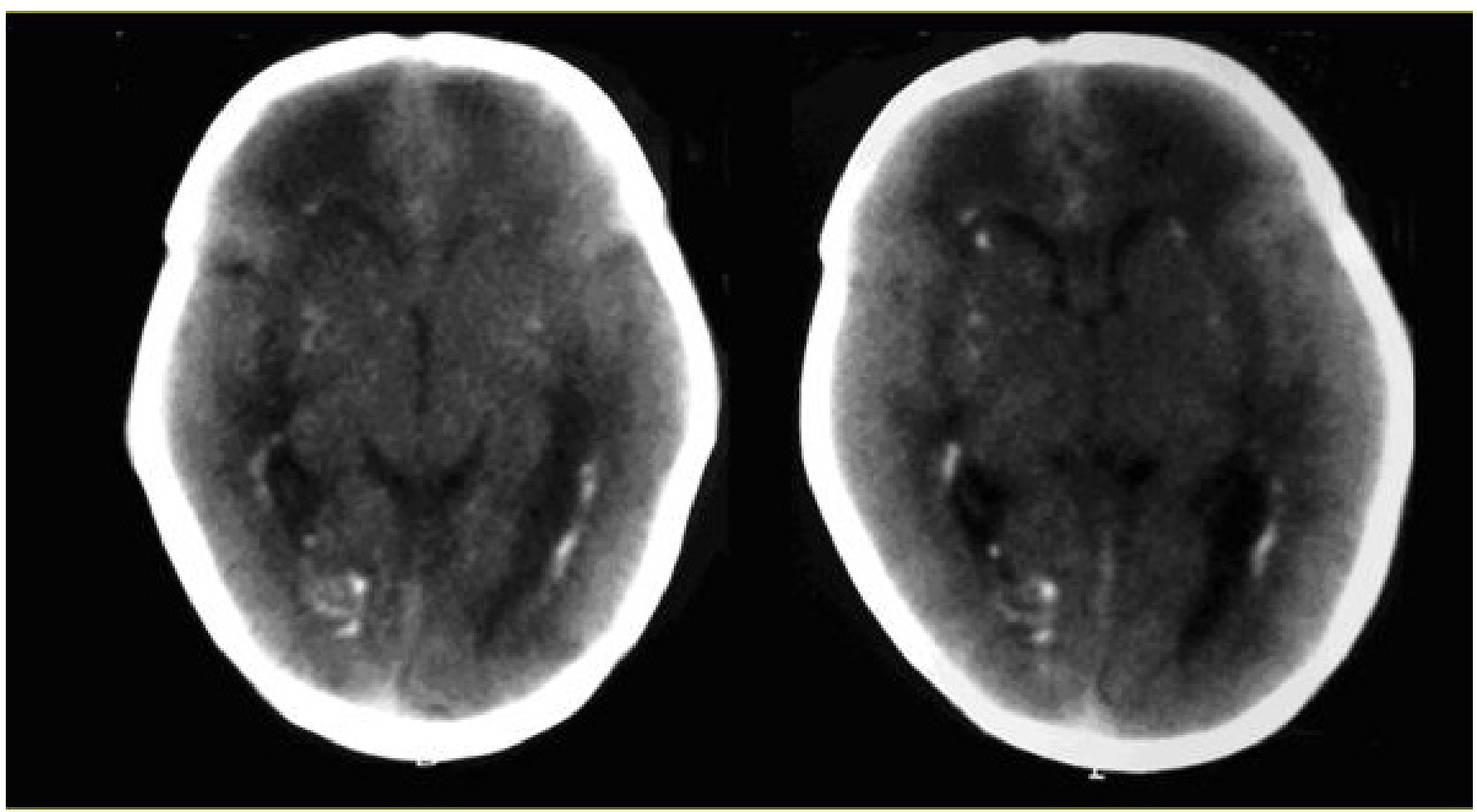

Tomography of the skull with periventricular calcifications commonly found in cytomegalovirus

A female infant born at 37 weeks and 2 days of gestation by vaginal delivery was admitted to the neonatal intensive care unit on July 11, 2009 with birth weight of $2,030 \mathrm{~g}$, height of $41,0 \mathrm{~cm}$ and head circumference of $31.0 \mathrm{~cm}$. The infant Apgar were 8, 9 and 9 at 1, 5 and $10 \mathrm{~min}$, respectively. A diagnosis of congenital toxoplasmosis was entertained because of the mother clinical history (she IgG positive and IgM positive for toxoplasmosis with avidity of $85 \%$ during first trimester of pregnancy). At the first trimester morphology scan intracranial calcifications were seen. The newborn had intrauterine growth restriction, splenomegaly, petechias and thrombocytopenia. Treatment of toxoplasmosis was done with sulphadiazine, pyrimethamine and folinic acid. The tomography of the skull showed multiple foci of bilateral periventricular calcifications and on the base nuclei. Due to these findings the serology for cytomegalovirus of mother and child was ordered. The mother was IgM negative, but IgG positive. The newborn was $\operatorname{IgM}$ and $\operatorname{IgG}$ positive. Cytomegalic inclusions in newborn's urine and polymerase chain reaction (PCR) for CMV had positive results. The newborn was discharged and was referred to outpatient clinic to be treated with ganciclovir.

The congenital infections, syphilis, toxoplamosis, rubella, cytomegalovirus and herpes correspond to

\footnotetext{
'Faculdade de Medicina, Universidade de Santo Amaro - UNISA, São Paulo (SP), Brazil.

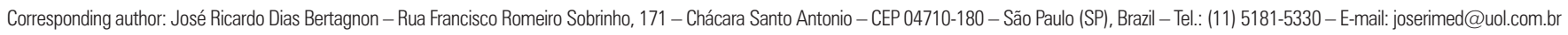
Received on: March 10, 2011 - Accepted on: Aug 8, 2011
} 
the acronym STORCH, and are characterized by common symptoms as: intrauterine growth restriction, hepatosplenomegaly, jaundice with increased direct bilirubin, anemia, petechiae, skin and mucosa lesions, encephalitis, pneumonia and thrombocytopenia ${ }^{(1,2)}$. To diagnosis STORCH the most common methods are serological research of IgM and IgG, PCR and imaging exams. The clinical picture of toxoplamosis mainly shows hydrocephalus or microcephaly, diffuse cerebral calcifications in cerebral cortex, microphthalmia and chorioretinitis (due to microcephaly) ${ }^{(3)}$. The cytomegalovirus causes periventricular calcifications, and in ophthalmoscopy the chorioretinitis is not associated to microphthalmia. Herpes is identified when maternal genital herpes is diagnosed.

To make a distinction among congenital infections that comprise the STRORCH complex, which leads to a specific diagnosis, the maternal clinical history,and events during gestation must be investigated, including laboratory tests.

In newborns some unspecific tests could be done such as hemogram with platelet, bilirubin, cerebrospinal fluid,as well as specific tests such as quantitative IgM, specific Igm for syphilis, toxoplamosis, rubella and cytomegalovirus. There is also a chance to investigate cytomegalic inclusions in urine, by skeletal radiography (skull, chest, long bones in the first week and if needed to repeat it in the second month), and by ophthalmoscopy.

The tomography of the skull is fundamental to clarify the diagnosis of cytomegalovirus ${ }^{(4)}$ since maternal clinical history, gestational ultrasound and clinical exam of newborn may suggest the diagnosis of congenital toxoplasmosis.

\section{REFERENCES}

1. Brock R, Segre CA. Doença de inclusão citomegálica. In: Segre CA. Perinatologia: fundamentos e práticas. São Paulo: Sarvier; 2002. p. 139-44.

2. Kinney JS, Kumar ML. Should we expand the TORCH complex? A description of clinical and diagnostic aspects of selected old and new agents. Clin Perinatol. 1988;15(4):727-44.

3. Rawlinson WD. Broadsheet. Number 50: Diagnosis of human cytomegalovirus infection and disease. Pathology. 1999;31 (2):109-15.

4. Mahboubi S. Radiological findings in perinatal infections. Clin Perinatol. 1981;8(3):517-36. 